\title{
Sema7A Signaling is Essential for Activity-Dependent Synapse Formation in the Mouse Olfactory Bulb
}

\author{
Nobuko Inoue ${ }^{1,2}$, Hitoshi Sakano ${ }^{2 *}$
}

'Department of Biophysics and Biochemistry, Graduate School of Science, The University of Tokyo, 2-11-16 Yayoi, Bunkyo-ku, Tokyo $113-0032$, Japan ${ }^{2}$ Department of Brain Function, University of Fukui School of Medicine, 23-3 Shimo-aizuki, Matsuoka, Fukui 910-1193, Japan

\section{Article Info}

\section{Article Notes}

Received: September 12, 2018

Accepted: October 12, 2018

\section{*Correspondence:}

Dr. Hitoshi Sakano, Department of Brain Function, University of Fukui School of Medicine, 23-3 Shimo-aizuki, Matsuoka, Fukui 910-1193, Japan; E-mail: sakano.hts@gmail.com.

(c) 2018 Sakano $\mathrm{H}$. This article is distributed under the terms of the Creative Commons Attribution 4.0 International License

\begin{abstract}
Odorant molecules are detected by olfactory sensory neurons (OSNs) in the olfactory epithelium. Odor information is then transmitted to the olfactory cortex through synapses with mitral/tufted (M/T) cells. We recently analyzed a pair of signaling molecules, Semaphorin (Sema) 7A expressed in OSN axons and its receptor Plexin (Plxn) C1 localized to M/T-cell dendrites. In the knockout mice for Sema7A or PlxnC1, initiation of synapse formation is perturbed. Rescue and reconstitution experiments demonstrated that interactions of Sema7A and PlxnC1 are essential to induce the post-synaptic assembly. Pharmacological blocking of NMDA receptors indicated that synaptic transmission induces primary-dendrite selection after the synapses are formed. We conclude that Sema7A/PIxnC1 signaling is key for initiating synapse formation followed by dendrite selection in $M / T$ cells. Since other Sema molecules are known to regulate targeting of OSN axons without involving neuronal activities, Sema7A is a unique example of Sema family proteins that regulates synapse formation and dendrite selection in an activity-dependent manner. Possible roles of Sema7A/PIxnC1 signaling will be discussed in the context of olfactory circuit formation in neonates.
\end{abstract}

\section{Introduction}

The mammalian olfactory system can detect a variety of odors using approximately 1,000 odorant receptor (OR) species ${ }^{1}$. It is wellestablished that each olfactory sensory neuron (OSN) expresses one functional OR gene in a mono-allelic manner ${ }^{2-5}$. Furthermore, OSN axons expressing the same type of OR converge to a specific target site, glomerulus, in the olfactory bulb $(\mathrm{OB})^{6-8}$. As a given odorant interacts with multiple OR species and a given OR responds to multiple odorants ${ }^{2}$, binding signals of odor molecules detected in the olfactory epithelium (OE) are converted to a unique set of activated glomeruli, a odor map, in the $\mathrm{OB}^{7,9,10}$. This conversion enables the brain to detect and discriminate a large repertoire of odorants ${ }^{11}$.

Since the discovery of OR genes in 1991 by Buck and Axel ${ }^{1}$, it has well been studied how OSN axons target to the OB for glomerular map formation ${ }^{12-25}$. During development, a coarse olfactory map is generated by a combination of dorsal-ventral (D-V) projection and anteriorposterior (A-P) projection. $\mathrm{D}-\mathrm{V}$ projection is regulated by positional information of OSNs in the $\mathrm{OE}^{12-19}$, while A-P projection is instructed by OR molecules using cAMP as a second messenger ${ }^{20,21}$. Different sets of Neuropilin (Nrp), Semaphorin (Sema), and Plexin (Plxn) molecules are known to regulate these processes ${ }^{19,21-25}$ : A-P projection is regulated by Nrp1 and Sema3A ${ }^{26}$, and D-V projection is by Nrp2 and Sema3F ${ }^{19}$. In addition to these targeting processes that occur in an activityindependent manner, olfactory circuits are further refined by OSN 
activity. Multiple sets of axon sorting molecules whose expression is regulated by OR-derived OSN activity, are responsible for glomerular segregation ${ }^{27}$.

Although primary projection autonomously takes place $^{26}$ even in the absence of the target $\mathrm{OB}^{28}$, proper connections are needed with mitral/tufted (M/T) cells in the $\mathrm{OB}$ to make the olfactory circuit functional. For synapse formation, pairing of OSN axons with nearby M/T cells takes place and then post-synaptic events are initiated in $\mathrm{M} / \mathrm{T}$-cell dendrites. Once the synapse is formed, primary dendrites are selected in a competitive manner. Synaptic activity is assumed to have a critical role in stabilizing the dendrite structure ${ }^{29}$. In contrast to the targeting of OSN axons, little was known about the events that lead to the formation of synapses with M/T-cell dendrites and a number of important questions were to be answered. For example, what kind of molecules mediate triggering of synapse formation and how are the synaptic structures selected and maintained in an activity-dependent manner? In an effort to address these questions, we recently studied signaling molecules, Sema7A and its receptor PlxnC1, essential for synapse formation and dendrite selection in $\mathrm{M} / \mathrm{T}$ cells ${ }^{30}$. In this mini-review, we will briefly summarize our recent work on Sema7A/PlxnC1 signaling in the mouse olfactory system.

\section{Sema7A Expressed in OSN Axons}

Various molecules, e.g., Neurexins/Neuroligins, ephrin/ Eph receptors, and Teneurins, are known to be involved in synaptic connections and circuit formation in various systems ${ }^{31-36}$. In the mammalian olfactory system, however, it is poorly understood how the OSN axons are connected with M/T-cell dendrites for synapse formation. How are these two types of cells matched, and what kind of signaling molecules are essential for triggering the post-synaptic events within glomeruli? We searched for a receptor and ligand pair expressed in OSN axons and M/T-cell dendrites in neonates. Almost forty axon guidance, cell adhesion, and signaling molecules were analyzed for their expression in the neonate $\mathrm{OE}$ and $\mathrm{OB}$. Among them, we found a promising pair, Sema7A and its receptor PlxnC1 expressed in OSN axons and M/T-cell dendrites, respectively ${ }^{30}$. Sema7A is a membrane-bound, glycosyl-phosphatidylinositol anchor protein $^{37}$, functioning in the immune and central nervous systems $^{38}$. Our immuno-electron microscopic study revealed that Sema7A is indeed localized to the pre-synaptic axon terminal of OSNs. It was found that expression levels of Sema7 A vary among different glomeruli. This indicates that the Sema7A gene is regulated by the OR-derived neuronal activity as found for glomerular segregation molecules ${ }^{27}$. Activity dependency of Sema7A expression was confirmed by analyzing of mutant mice lacking CNG-A2, a component of cyclic nucleotide-gated (CNG) channels ${ }^{39}$. We also analyzed the glomerulus expressing the DRY-motif mutant-
OR that was unable to couple with $\mathrm{G}$ proteins ${ }^{21}$. It was found that expression levels of Sema7A were significantly reduced in the glomeruli expressing the G-protein coupling mutant of $\mathrm{OR}^{30}$.

What is in vivo function of Sema7A within glomeruli? We generated and analyzed the Sema7A conditionalknockout (cKO) specific to OSNs expressing rI7 receptor ${ }^{30}$. Since the KOs of other Sema molecules severely affect targeting of OSN axons ${ }^{19,}{ }^{21-25}$, we examined whether glomerular map was normally formed in the Sema7A cKO. Close examination of rI7-expressig OSNs did not reveal any sign of impairment in targeting of OSNs to the OB. However, when M/T cells were analyzed, synapse formation and dendrite selection were perturbed. In the Sema7A total knockout (KO) ${ }^{40}$, the synapse number stayed low and post-synaptic density (PSD) was rarely found in the M/T-cell dendrites. We then examined preand post-synaptic markers in the synapses of Sema7A cKO and total KO. Immunostaining revealed that both markers were markedly reduced in these KOs. We also analyzed dendrite morphology of $\mathrm{M} / \mathrm{T}$ cells in the Sema7A cKO by injecting Lucifer Yellow into the rI7 glomeruli. Unlike in the WT, dendrites remained branched and multiple dendrites were often observed for single M/T cells in the cKO. This observation indicates that Sema7A expressed by OSNs plays important roles not only in inducing postsynaptic events but also in selecting primary dendrites of $\mathrm{M} / \mathrm{T}$ cells.

\section{PlxnC1 Localized to M/T-cell Dendrites}

We next studied how the Sema7A signal is processed in $\mathrm{M} / \mathrm{T}$ cells. PlxnC1 is known to serve as a receptor for Sema7A in other systems ${ }^{40,41}$. In Purkinje cells, Sema7A was reported to facilitate synapse elimination of mossy fibers via PlxnC $1^{42}$. In our study, immunohistochemistry of $\mathrm{OB}$ sections detected accumulation of PlxnC1 in the glomerular layer, indicating that PlxnC1 produced by $\mathrm{M} / \mathrm{T}$ cells is localized to their tuft structures of dendrites within glomeruli. It should be noted that PlxnC1 is detected in the M/T-cell dendrites only during the early neonatal period. Since both Sema7A and PlxnC1 are expressed in the membrane-bound form, it is likely that they directly interact with each other and function as signaling molecules to initiate synapse formation. In order to study the in vivo function of PlxnC1, we generated the $\mathrm{M} / \mathrm{T}$-cell-specific cKO for PlxnC1. In the $\mathrm{OB}, \mathrm{M} / \mathrm{T}$-cell migration and dendrite extension occurred normally in the PlxnC1 cKO, and targeting of OSN axons was also normal. However, initial assembly of synaptic structures was impaired and dendrite selection was delayed as seen in the Sema7A KOs whose impairments remained into adulthood. These results demonstrate that PlxnC1 is also needed to initiate the synapse formation and dendrite selection in $\mathrm{M} / \mathrm{T}$ cells. 


\section{Post-synaptic Events Induced by Sema7A-PIxnC1 Interaction}

How does the Sema7A-PlxnC1 interaction induce the post-synaptic events? We studied PSD formation triggered by Sema7A using the heterologous system in vitro. The secrete-type of Sema7A and myc-tagged PlxnC1 genes were introduced into HEK293 cells in culture, where the secreted Sema7A interacts with PlxnC1 expressed on the HEK-cell surface. To analyze the aggregation of PSD, cells had been transfected with the FLAG-tagged gene for SAP90 that serves as a scaffold molecule in clustering the PSD ${ }^{32,43}$, ${ }^{44}$. PSD formation is the earliest hallmark of post-synaptic events in M/T-cell dendrites ${ }^{32,45,46}$. Co-localization of SAP90 with PlxnC1 was found by immunostaining of FLAG and myc tags, when the full-length PlxnC1 was expressed with the secrete type of Sema7A. In contrast, with the mutant PlxnC1 deleting the cytosolic domain, co-localization of SAP90 was not seen. We then examined whether the Sema7A-PlxnC1 interaction is indeed essential for triggering PSD formation. Based on the three-dimentional analysis of Sema7 $\mathrm{A}^{47}$, we introduced the amino acid substitution, $\mathrm{Y} \rightarrow \mathrm{S}$ at residue 213 , into the interaction site of Sema7A with PlxnC1. In our experiment, co-localization of SAP90 with PlxnC1 was not induced with the mutant Sema7A. This observation further supports the idea that PlxnC1 serves as a receptor for Sema7A in its interaction between OSN axons and M/Tcell dendrites. Physical interactions between Sema7A and PlxnC1 were also confirmed by using fusion proteins with alkaline phosphatase. These results demonstrate that Sema7A/PlxnC1 signaling recruits SAP90, and that the Sema7A-PlxnC1 interaction is essential for triggering postsynaptic events in M/T cells (Figure 1A).

As mentioned earlier, it is not only the initiation of synapse formation, but also primary dendrite selection that is perturbed in the Sema7A KOs and PlxnC1 cKO. This is probably because the trans-synaptic activity from OSN axons is needed for dendrite selection in M/T cells after the establishment of functional synapses. To examine this, we used an NMDA-R inhibitor, MK801, to pharmacologically inhibit $\mathrm{Ca}^{2+}$ entry into $\mathrm{M} / \mathrm{T}$ cells ${ }^{48}$. This treatment blocks synaptic activity after synapses have been formed. In our experiment, transcripts of the cpg15 gene, a marker for synaptic activity ${ }^{49}$, were measured in M/T cells after MK801 treatment. We found that MK801 significantly reduced $\operatorname{cpg} 15$ expression and suppressed dendrite selection. These results indicate that synaptic transmission, but not Sema7A/PlxnC1 signaling directly, promotes synaptic competition, causing dendrite selection and branch pruning in $\mathrm{M} / \mathrm{T}$ cells (Figure 1B).

\section{Discussion}

Since the discovery of OR genes ${ }^{1}$, many groups have studied OR-instructed axonal projection of OSNs, and major processes of glomerular map formation have been

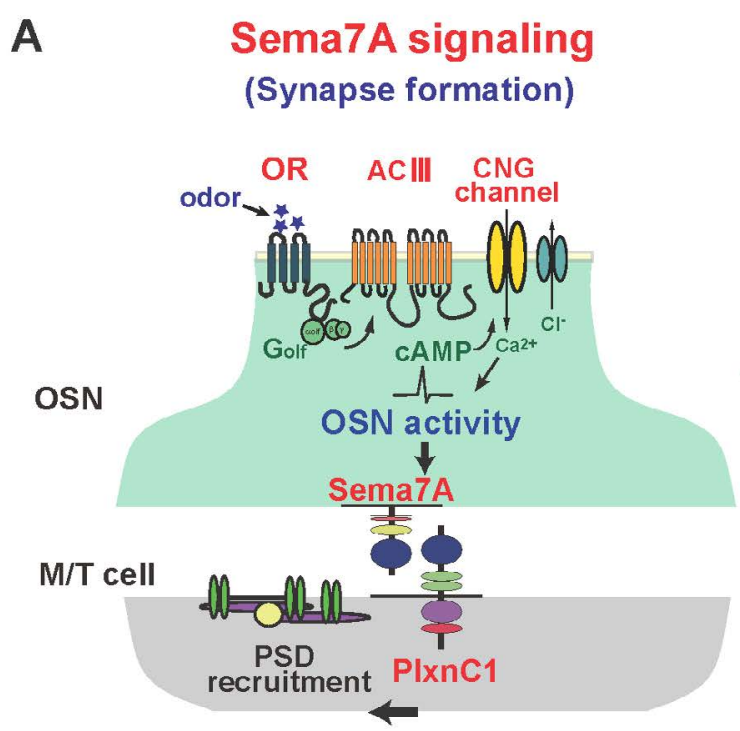

B

\section{Trans-synaptic activity \\ (Dendrite selection)}

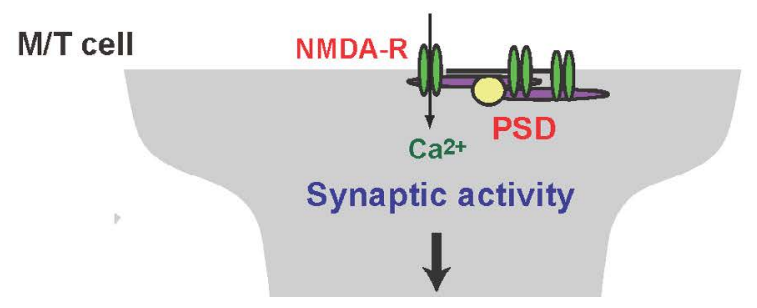

Figure 1: Schematic diagrams of synapse formation and dendrite selection regulated by Sema7A/PIxnC1 signaling.

(A) Sema7A/PlxnC1 signaling is essential for triggering post-synaptic events in $M / T$ cells. OR-derived neuronal activity of OSNs induces Sema7A expression in OSN axons. Sema7A interacts with its receptor PlxnC1 localized in M/T-cell dendrites and recruits PSD molecules.

(B) Synaptic transmission promotes synaptic competition, causing dendrite selection in $\mathrm{M} / \mathrm{T}$ cells. After establishing synapses between OSN axons and $\mathrm{M} / \mathrm{T}$-cell dendrites, trans-synaptic activities from OSNs induce dendrite selection and branch pruning in $\mathrm{M} / \mathrm{T}$ cells.

elucidated $^{12-27}$. In contrast, olfactory circuit formation mediated by the secondary neurons, M/T cells, has long been elusive. What kind of molecules are involved in synapse formation with OSNs, and how is targeting of M/T cells regulated to various areas in the OC? Our recent studies revealed that interactions between Sema7A expressed in OSN axons and PlxnC1 in M/T-cell dendrites are essential for triggering synapse formation within glomeruli. Since other Sema molecules in the olfactory system are known to regulate targeting of OSN axons independently from neuronal activity ${ }^{19,} 21-25$, Sema7A described in our study is a unique example of Sema family proteins, which triggers synapse formation in an activity-dependent manner. 
We found that the interaction of Sema7A with PlxnC1 induces postsynaptic events within glomeruli by recruiting SAP90 to assemble the PSD. Then, how is it that Sema7A/ PlxnC1 signaling recruits PSD molecules? One possibility is a conformational change in PlxnC $1^{47}$ caused by binding of Sema7A to PlxnC1. This may induce the tyrosine phosphorylation that allows the assembly of PSD molecules. It is also possible that Sema7A-PlxnC1 interactions activate signal transduction pathways. Recent studies demonstrate that transmembrane molecules induce PSD formation by their own kinase activity, and not by recruiting PSD molecules via PSD domains ${ }^{50}$. A most likely scenario is that Sema7A/PlxnC1 signaling activates the PAK-Rac1/Cdc42 pathway $^{30,51,52}$ which in turn regulates vesicle trafficking, cytoskeletal dynamics, and gene expression necessary for the post-synaptogenesis in M/T-cell dendrites. Then, how does pre-synaptic maturation occur in primary axons? As GPI-anchored Sema7A has no cytosolic domain in OSNs, it is unlikely that Sema7A signaling directly induces maturation of pre-synaptic structures. We assume that PSD-maturation induces pre-synaptic formation in a retrograde manner by using other membrane proteins or permeable factors.

These processes in both pre- and post-synaptic events need to be clarified in the future.

It is interesting that Sema7A expression is enhanced by odorous ligands during the neonatal period. When pups are exposed to vanillin, a ligand to MOR29A ${ }^{53}$, Sema7A expression is increased and synapse formation is enhanced in the MOR29A glomeruli. Environmental odors, e.g., maternal smell, may help pups to establish specific neural circuits by up-regulating Sema7A expression for their survival. Activity-dependent expression of Sema7A may be beneficial for the adaptation of pups to the postnatal environment during olfactory circuit formation. Another intriguing possibility is olfactory imprinting during the critical period. It is possible that enhanced odor inputs mediated by Sema7A signaling may help establish the imprinted odor memory at early neonatal stages. These possibilities need to be studied in the future.

In the mammalian olfactory system, primary neurons are constantly regenerated replacing old OSN axons with new ones. In contrast, secondary neurons, $\mathrm{M} / \mathrm{T}$ cells, are not replaced and glomerular structures are kept stably throughout the lifetime ${ }^{54,55}$. It is interesting to study how the synapses are regenerated in adults using pre-existing glomerular structures. In the neuromuscular junction, the post-synaptic structure is maintained without the pre-synapse ${ }^{56}$, and interneurons help the post-synaptic structures to survive ${ }^{57}$. Synapse formation of regenerating OSN axons may be regulated in a different manner than that in the neonatal animal. How do the regenerating OSN axons find their target glomeruli in adults? Do they follow the pre-existing axons of OSNs expressing the same type of
OR? These studies on olfactory map regeneration will give us a new insight into our understanding of the olfactory disfunction Parosmia caused by physical damage that destroys the wiring of OSN axons.

In our study, we described two types of neuronal activities: one is the OR-derived activity that regulates Sema7A expression in OSN axons (Figure 1A) and the other is trans-synaptic activity for dendrite selection in $\mathrm{M} / \mathrm{T}$ cells (Figure 1B). In the mouse olfactory system, proper olfactory circuits may be established by selecting primary dendrites of $\mathrm{M} / \mathrm{T}$ cells in an activity-dependent manner. It is interesting to further investigate whether $\mathrm{M} / \mathrm{T}$ cells are naïve with respect to circuit formation that drive innate olfactory behaviors. It is important to determine how much of such processes are genetically programmed and how much can be modulated by environmental inputs. These interesting questions are expected to be answered in the future. The mouse olfactory system will continue serving as an excellent model system for the study of decision making and memory formation in the mammalian brain.

\section{Acknowledgements}

We would like to thank H. Nishizumi, H. Naritsuka, and H. Kiyonari for their collaboration in this study. This work was supported by a Specially Promoted Research Grant and grants in aid from MEXT of Japan. N. I. was a pre-doctoral fellow of the Japan Society for the Promotion of Science and supported by Japan Foundation for Applied Enzymology.

\section{References}

1. Buck LB, Axel R. A novel multigene family may encode odorant receptors: a molecular basis for odor recognition. Cell. 1991; 65: 175187.

2. Malnic B, Hirono J, Sato T, et al. Combinatorial receptor codes for odors. Cell. 1999; 96: 713-723.

3. Serizawa S, Ishii T, Nakatani $\mathrm{H}$, et al. Mutually exclusive expression of odorant receptor transgenes. Nat. Neurosci. 2000; 3: 687-693.

4. Chess A, Simon I, Cedar H, et al. Allelic inactivation regulates olfactory receptor gene expression. Cell. 1994; 78: 823-834.

5. Ishii T, Serizawa S, Kohda A, et al. Monoallelic expression of the odourant receptor gene and axonal projection of olfactory sensory neurons. Genes Cells. 2001; 6: 71-78.

6. Mombaerts P, Wang F, Dulac C, et al. Visualizing an olfactory sensory map. Cell. 1996; 87: 675-686.

7. Ressler KJ, Sullivan SL, Buck LB. Information coding in the olfactory system: evidence for a stereotyped and highly organized epitope map in the olfactory bulb. Cell. 1994; 79: 1245-1255.

8. Vassar R, Chao SK, Sitcheran R, et al. Topographic organization of sensory projections to the olfactory bulb. Cell. 1994; 79: 981-991.

9. Buck LB. Information coding in the vertebrate olfactory system. Annu. Rev Neurosci. 1996; 19: 517-544.

10. Mori K, Yoshihara Y. Molecular recognition and olfactory processing in the mammalian olfactory system. Prog Neurobiol. 1995; 45: 585-619.

11. Mori K, Sakano H. How is the olfactory map formed and interpreted in the mammalian brain. Annu. Rev Neurosci. 2011; 34: 467-499. 
12. Alenius M, Bohm S. Identification of a novel neural cell adhesion molecule-related gene with a potential role in selective axonal projection. J Biol Chem. 1997; 272: 26083-26086.

13. Miyamichi K, Serizawa S, Kimura HM, et al. Continuous and overlapping expression domains of odorant receptor genes in the olfactory epithelium determine the dorsal/ventral positioning of glomeruli in the olfactory bulb. J Neurosci. 2005; 25: 3586-3592.

14. Ressler KJ, Sullivan SL, Buck LB. A zonal organization of odorant receptor gene expression in the olfactory epithelium. Cell. 1993; 73: 597-609.

15. Saucier D, Astic L. Analysis of the topographical organization of olfactory epithelium projections in the rat. Brain Res Bull. 1986; 16: 455-462.

16. Strotmann J, Wanner I, Helfrich T, et al. Rostrocaudal patterning of receptor-expressing olfactory neurons in the rat nasal cavity. Cell Tissue Res. 1994; 278: 11-20.

17. Vassar R, Ngai J, Axel R. Spatial segregation of odorant receptor expression in the mammalian olfactory epithelium. Cell. 1993; 74: 309-318.

18. Yoshihara Y, Kawasaki M, Tamada A, et al. OCAM: A new member of the neural cell adhesion molecule family related to zone-to-zone projection of olfactory and vomeronasal axons. J Neurosci. 1997; 17: 5830-5842.

19. Takeuchi H, Inokuchi K, Aoki M, et al. Sequential Arrival and Graded Secretion of Sema3F by Olfactory Neuron Axons Specify Map Topography at the Bulb. Cell. 2010; 141: 1056-1067.

20. Chesler AT, Zou DJ, Le Pichon CE, et al. A G protein/cAMP signal cascade is required for axonal convergence into olfactory glomeruli. Proc Natl Acad Sci USA. 2007; 104: 1039-1044.

21. Imai T, Suzuki M, Sakano H. Odorant receptor-derived cAMP signals direct axonal targeting. Science. 2006; 314: 657-661.

22. Walz A, Rodriguez I, Mombaerts P. Aberrant sensory innervation of the olfactory bulb in neuropilin-2 mutant mice. J Neurosci. 2002; 22: 4025-4035.

23. Taniguchi M, Nagao H, Takahashi YK, et al. Distorted odor maps in the olfactory bulb of semaphorin 3A-deficeint mice. J Neurosci. 2003; 23 1390-1397.

24. Schwarting GA, Raitcheva D, Crandall JE, et al. Semaphorin 3A-mediated axon guidance regulates convergence and targeting of P2 odorant receptor axons. Eur J Neurosci. 2004; 19: 1800-1810.

25. Cloutier JF, Sahay A, Chang EC, et al. Differential requirements for semaphorin $3 \mathrm{~F}$ and Slit-1 in axonal targeting, fasciculation, and segregation of olfactory sensory neuron projections. J Neurosci. 2004; 24: 9087-9096.

26. Imai T, Yamazaki T, Kobayakawa R, et al. Pre-target axon sorting establishes the neural map topography. Science. 2009; 325: 585-590.

27. Serizawa S, Miyamichi K, Takeuchi $\mathrm{H}$, et al. A neuronal identity code for the odorant receptor-specific and activity-dependent axon sorting. Cell. 2006; 127: 1057-1069.

28. St John JA, Clarris HJ, McKeown S, et al. Sorting and convergence of primary olfactory axons are independent of the olfactory bulb. J Copm Neurol. 2003; 464: 131-140

29. Cline $\mathrm{H}$, Haas $\mathrm{K}$. The regulation of dendritic arbor development and plasticity by glutamatergic synaptic input: a review of the synaptotrophic hypothesis. J Physiol. 2008; 586: 1509-1517.

30. Inoue N, Nishizumi $\mathrm{H}$, Naritsuka $\mathrm{H}$, et al. Sema7A/PlxnC1 signaling triggers activity-dependent olfactory synapse formation. Nat Commun. 2018; DOI: 10.1038.

31. Südhof TC. Neuroligins and neurexins link synaptic function to cognitive disease. Nature. 2008; 455: 903-911.

32. Siddiqui TJ, Craig AM. Synaptic organizing complexes. Curr Opin Neurobiol. 2011; 21: 132-143.

33. Hruska M, Dalva MB. Ephrin regulation of synapse formation, function and plasticity. Mol Cell Neurosci. 2012; 50: 35-44.

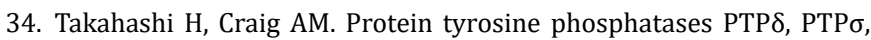
and LAR: presynaptic hubs for synapse organization. Trends Neurosci. 2013; 36: 522-534.

35. Mosca TJ. On the teneurin track: a new synaptic organization molecule emerges. Front Cell Neurosci. 2015; 9: 1-14.

36. de Wit J, Ghosh A. Specification of synaptic connectivity by cell surface interactions. Nat Rev Neurosci. 2016; 17: 4.

37. Martin G. The glycosyl-phosphatidylinositol anchor of membrane proteins. Btochimica et Biophysica Acta. 1989; 988: 427-454.

38. Alto LT, Terman JR. Semaphorins and their Signaling Mechanisms. Methods Mol Biol. 2017; 1493: 1-25.

39. Lin DM, Wang F, Lowe G, et al. Formation of precise connections in the olfactory bulb occurs in the absence of odorant-evoked neuronal activity. Neuron. 2000; 26: 69-80.

40. Pasterkamp RJ, Peschon JJ, Spriggs MK, et al. Semaphorin 7A promotes axon outgrowth through integrins and MAPKs. Nature. 2003; 424: 398-405.

41. Tamagnone L, Artigiani S, Chen H, et al. Plexins are a large family of receptors for transmembrane, secreted, and GPI-anchored semaphorins in vertebrates. Cell. 1999; 99: 71-80.

42. Uesaka N, Uchigashima M, Mikuni T, et al. Retrograde semaphorin signaling regulates synapse elimination in the developing mouse brain. Science. 2014; 344: 1020-1023.

43. Burkhardt C, Müller M, Badde A, et al. Semaphorin 4B interacts with the post-synaptic density protein PSD-95/SAP90 and is recruited to synapses through a C-terminal PDZ-binding motif. FEBS Lett. 2005; 579: 3821-3828.

44. Chen SY, Cheng HJ. Functions of axon guidance molecules in synapse formation. Curr Opin Neurobiol. 2009; 19: 471-478.

45. Frank R, Grant S. Supramolecular organization of NMDA receptors and the postsynaptic densitiy. Curr Opin Neurobiol. 2015; 45: 139-147.

46. Favuzzi E, Rico B. Molecular diversity underlying cortical excitatory and inhibitory synapse development. Curr Opin Neurobiol. 2018; 53: 8-15.

47. Liu H, Juo ZS, Shim AH, et al. Structural Basis of Semaphorin-Plexin Recognition and Viral Mimicry from Sema7A and A39R Complexes with PlexinC1. Cell. 2010; 142: 749-761.

48. Woodruff GN, Foster AC, Gill R, et al. The interaction between MK-801 and receptors for $\mathrm{N}$-methyl-D-aspartate: functional consequences. Neuropharmacol. 1987; 26: 903-909.

49. Nedivi E, Wu GY, Cline HT. Promotion of dendritic growth by CPG15, an activity-induced signaling molecule. Science. 1998; 281: 1863-1866.

50. Missler M, Südhof TC, Biederer T. Synaptic Cell Adhesion. C S H Persp in Biol. 2012; 4: 1-18.

51. Boda B, Dubos A, Muller D. Signaling mechanisms regulating synapse formation and function in mental retardation. Curr Opin Neurobiol. 2010; 20: 519-527.

52. Negishi M, Oinuma I, Katoh H. Plexins: axon guidance and signal transduction. Cell Mol Life Sci. 2005; 62: 1363-1371.

53. Tsuboi A, Imai T, Kato HK, et al. Two highly homologous mouse odorant receptors encoded by tandemly linked MOR29A and MOR29B genes differently respond to phenyl ethers. Eur J Neurosci. 2011; 33: 205-213. 
54. Mizrahi A, Katz LC. Dendritic stability in the adult olfactory bulb. Nat Neurosci. 2003; 6: 1201-1207.

55. Chen WR, Shepherd GM. The olfactory glomerulus: a cortical module with specific functions. J Neurocytol. 2005; 34: 353-360.

56. Vaughn JE, Barber RP, Sims TJ. Dendritic development and preferential growth into synaptogenic fields: A quantitative study of Golgiimpregnated spinal motor neurons. Synapse. 1988; 2: 69-78.

57. Willmann R, Pun S, Stallmach L, et al. Cholesterol and lipid microdomains stabilize the postsynapse at the neuromuscular junction. EMBO J. 2006; 25: 4050-4060. 